\title{
Derrida, Kittler et le calendrier des post-
}

\section{Antonin Wiser et Katia Schwerzmann}

\section{(2) OpenEdition}

Journals

Édition électronique

URL : http://journals.openedition.org/edl/2356

DOI : 10.4000/edl.2356

ISSN : 2296-5084

\section{Éditeur}

Université de Lausanne

\section{Édition imprimée}

Date de publication : 15 mars 2020

Pagination : 75-80

ISBN : 978-2-940331-73-4

ISSN : 0014-2026

\section{Référence électronique}

Antonin Wiser et Katia Schwerzmann, « Derrida, Kittler et le calendrier des post- », Études de lettres [En ligne], 312 | 2020, mis en ligne le 24 mars 2020, consulté le 17 septembre 2020. URL : http:// journals.openedition.org/edl/2356 ; DOI : https://doi.org/10.4000/edl.2356 


\section{DERRIDA, KITTLER ET LE CALENDRIER DES POST-}

Au commencement, en principe, était la poste.

Jacques Derrida

1980 : La carte postale de Jacques Derrida s'ouvre par des "Envois» au pluriel. Sans adresse identifiable (sinon la virtuosité de leur auteur), ils sont ainsi mis en circulation comme autant de bouteilles à la mer, après bien d'autres que la déconstruction lançait depuis 15 ans par-dessus bord pour se disséminer à travers le monde. Échouant sur divers rivages, elle connut les belles réussites que l'on sait - outre-Atlantique -, et que l'on sait moins - outre-Rhin. Parmi ceux quatteignirent les missives ainsi hasardées, il s'en trouva qui voulurent engager une correspondance intellectuelle avec celui qui signait les envois de son nom propre. Que certaines de leurs réponses n'arrivassent jamais à destination, voilà qui devait arriver - si «la condition pour que ça arrive, c'est que ça finisse et même que ça commence par ne pas arriver» ${ }^{1}$.

1980 : Erich Brinkmann et Günter Bose fondent à Berlin une petite maison d'édition qui publiera Die Postkarte en deux livraisons ("Envois" en 1982 et "Spekulieren über/auf 'Freud'» en 1987) dans la traduction de Hans-Joachim Metzger. Il s'agit de l'un des premiers textes de Derrida à être traduits en allemand (après Die Schrift und die Differenz et Die Stimme und das Phänomen, tous deux parus chez Suhrkamp respectivement en 1972 et 1979). Brinkmann \& Bose doivent d'avoir pu accueillir ce livre dans leur catalogue à la médiation d'un jeune chercheur associé de l'Université de Fribourg-en-Brisgau, Friedrich Kittler.

I. J. Derrida, La carte postale, p. 34. 
1980: un professeur allemand, alors directeur du Max-Planck-Institut à Starnberg, reçoit le prestigieux prix Adorno. Dans son discours de réception, intitulé "Die Moderne - ein unvollendetes Projekt", Jürgen Habermas prend les missives pour des missiles et, afin de conjurer un postmodernisme antimoderne nommément représenté par Derrida et Foucault, il convoquera «l'esprit de la modernité esthétique». La menace demeurant, il répétera l'opération au Collège de France lors d'une série de conférences ${ }^{2}$ destinées à renvoyer le colis piégé à son expéditeur. Pendant ce temps, c'est pourtant bien en Allemagne qu'on cherchait d'en finir avec l'esprit.

1980 : le jeune chercheur de Freiburg fait paraittre un ouvrage collectif dont le titre et la préface qu'il signe veulent faire entendre le tic-tac d'une bombe. Austreibung des Geistes aus den Geisteswissenschaften. Programme des Poststrukturalismus rassemble des textes d'auteurs américains, français et allemands, réunis par le "Zauberwort" ${ }^{3}$ latin de Studium Generale, titre du séminaire où ils furent invités à prononcer des conférences en 1978 et 1979. Jacques Derrida signe le premier texte, "Titel (noch zu bestimmen) / Titre (à préciser)», repris depuis lors dans Parages 4 .

$1800 / 1900 / 1980$ : dans sa préface, Kittler remonte la mécanique de l'engin explosif, une vieille montre assemblée lors de la réforme de l'éducation allemande entre 1770 et 1800 , où s'inaugurent les sciences humaines sous le signe de l'herméneutique. Au tournant du XXe siècle, on passe un cran, le ressort met en tension les prémisses d'un structuralisme qui ignore encore son nom, avec les travaux de Freud, Saussure et Frazer. Leurs successeurs donneront un tour de clé supplémentaire pour tenter de faire converger les trois discours de la psychanalyse, de la linguistique et de l'anthropologie. Mais vers 1980, la machine structuraliste éclate en son impossible unité: "Non seulement la juxtaposition d'hétérogénéités hétérogènes n'a aucun sens, mais elle fait sauter les structures. " ${ }^{5}$ C'est désormais l'heure des post-, et le recueil de Kittler compte y faire date sur la foi du cachet de son sous-titre, qui en annonce au pluriel les programmes. À l'instar de Habermas, d'aucuns verront

2. Rassemblées dans J. Habermas, Der philosophische Diskurs der Moderne.

3. F. Kittler, Austreibung des Geistes aus den Geisteswissenschaften, p. 13.

4. J. Derrida, Parages. Une note liminaire (p. 206) y rappelle la conférence de Fribourg et une première publication en 1981 dans la revue Nuova, mais passe sous silence la parution en allemand dans l'ouvrage dirigé par Kittler.

5. F. Kittler, Austreibung des Geistes aus den Geisteswissenschaften, p. 10. 
là un projet de timbrés puisqu'il s'agit à la lettre de perdre l'esprit en l'expulsant hors des Geisteswissenschaften.

1980: insistance du calendrier. On fomente outre-Rhin la stratégie destinée à faire fuir les vieux fantômes de l'herméneutique humaniste. Des alliances se nouent, on ajointe les forces. Kittler participe ainsi, avec Manfred Frank et Samuel Weber, à la création de la revue Fugen. Deutsch-Französisches Jahrbuch für Text-Analytik, qui entend favoriser la réception des philosophes français en Allemagne. Mais, selon le mot shakespearien dont Derrida fera dix ans plus tard le pivot de sa lecture des spectres de Marx, time is out of joint - die Zeit ist aus den Fugen: l'époque indisposée, la jonction franco-allemande échoue, et la revue ne connaitra pas d'autre numéro que celui qui paraît en 1980 .

1980-1986: Kittler poursuivra cependant le projet d'exorcisme formulé dans le titre de l'ouvrage collectif; et il le fera en travaillant le premier à l'élaboration d'une Medientheorie qui intègre les propositions de Derrida, Lacan et Foucault à une analyse systématique des supports d'enregistrement, des canaux de transmission, des dispositifs technologiques irréductibles au «sens» qu'ils font bien davantage que véhiculer:

Rien n'existe des individus, sinon ce que les média enregistrent et transmettent. Ce ne sont pas les messages ou les contenus, avec lesquels les techniques de communication équipent littéralement ce que l'on nomme des âmes pour la durée d'une époque technique, qui comptent, mais seulement [...] leurs circuits, ce schématisme de la perceptibilité 6 .

Ce faisant, le poststructuralisme du système postal derridien bascule vers le posthumanisme des courriers électroniques.

1980-2019: la Medientheorie kittlérienne a fait date, en effet; elle appartient désormais au corpus classique des instruments théoriques enseignés et étudiés dans les universités allemandes. Mais il semble que nous ne disposions encore ni d'une horloge mondiale ni d'un calendrier universel. L'histoire intellectuelle du monde francophone s'est poursuivie dans une ignorance quasi complète du devenir de ses propres envois extra muros. La réponse de Kittler aux bouteilles à la mer de la

6. F. Kittler, Gramophone, Film, Typewriter, p. 32. 
déconstruction est restée, en France, «lettre morte " ${ }^{7}$. L'année 1980 marque donc le point d'une occasion manquée, d'une rencontre inégale et non réciproque: deux temporalités s'y sont croisées sans parvenir à se synchroniser. Kittler a pris à la lettre le programme d'expulsion de l'esprit hors des sciences humaines dont il a perçu la promesse dans les œuvres des poststructuralistes parisiens, et il l'a poursuivi avec une originalité à laquelle la théorie française a fait la sourde oreille. Bien entendu, cette situation confirme l'hypothèse de La carte postale: une lettre peut toujours ne pas arriver à destination.

2019-... : c'est fort tardivement que l'on a retrouvé le colis allemand égaré. Quelques éditeurs et traducteurs s'efforcent aujourd'hui de le remettre en circulation posthume. Ainsi les esprits d'une lettre morte reviennent-ils post festum à l'expéditeur, après une longue différance: en provenance d'outre-tombe et ranimant ces auteurs qu'on a pu croire ici un peu vite enterrés. Il n'est pas sûr que nous sachions accuser bonne réception d'une réponse accusant tant d'indifférence hier, alors qu'on avait l'esprit ailleurs. Les effets de décalage, d'asynchronisme et de latence restent à ce jour difficilement calculables. Ce qui est en revanche certain, c'est que nous ne saurons jamais quelle histoire des post- aurait pu s'écrire si le programme kittlérien, avec ses machines conceptuelles et ses câblages médiumniques, avait trouvé à temps prise où se brancher en France.

Antonin Wiser

Section de français, Faculté des lettres, Université de Lausanne Katia Schwerzmann Program in Literature, Duke University

7. Voir K. Schwerzmann, "La lettre morte”". 


\section{BIBLIOGRAPHIE}

Derrida, Jacques, La carte postale, Paris, Flammarion, 1980.

—, Parages, Paris, Galilée, 1986-2003.

Habermas, Jürgen, Der philosophische Diskurs der Moderne. Zwölf Vorlesungen, Frankfurt am Main, Suhrkamp, 1985.

Kittler, Friedrich, Austreibung des Geistes aus den Geisteswissenschaften. Programme des Poststrukturalismus, Padeborn/München/Wien/ Zürich, Ferdinand Schöningh, 1980.

—, "Exorciser l'homme des sciences humaines. Programmes du poststructuralisme», trad. Slaven Waelti, Appareil, 19 (2017), en ligne: <https://journals.openedition.org/appareil/2522>.

-, Gramophone, Film, Typewriter (1986), trad. Frédérique Vargoz, Dijon, Les Presses du Réel, 2018.

Schwerzmann, Katia, "La lettre morte" - Friedrich Kittler en correspondance avec les poststructuralistes", Appareil, 19 (2017), en ligne: <https://journals.openedition.org/appareil/2552>. 
